Supporting Information for

\title{
Crumpled versus Flat Gold Nanosheets: Temperature-Regulated Synthesis and Their Plasmonic and Catalytic Properties
}

Gongguo Zhang, ${ }^{\mathrm{a}}$ Yanyun Ma, ${ }^{\mathrm{b}}$ Mengfan Li, ${ }^{\mathrm{c}}$ Shan Ren, ${ }^{\mathrm{b}}$ Xiaowei Fu, ${ }^{\mathrm{a}}$ Hongwen Huang, ${ }^{\mathrm{c}}$ and Yiqun Zheng*a

${ }^{a}$ Department of Chemistry and Chemical Engineering, Jining University, Qufu, Shandong 273115, P. R. China.

${ }^{b}$ Institute of Functional Nano \& Soft Materials (FUNSOM), Jiangsu Key Laboratory for CarbonBased Functional Materials \& Devices, Soochow University, Suzhou, Jiangsu 215123, P. R. China.

c School of Materials Science and Engineering, Hunan University, Changsha, Hunan 410082,

China.

${ }^{*}$ Corresponding Author: Prof. Y. Zheng, E-mail: whzyq@163.com

Number of page: 21; Number of figure: 15; Number of table: 3 


\section{Table of Content}

Figure S1. ED patterns of typical crumpled Au nanosheets.

Figure S2. Atomic-resolution HAADF-STEM images of crumpled Au nanosheet showing the presence of stepped facets on edges.

Figure S3. SEM images of Au products obtained via the standard procedure, except that the amount of Au precursor was reduced to a) $0.25 \mu \mathrm{mol}$ and b) $0.5 \mu \mathrm{mol}$, respectively.

Figure S4. SEM images showing effect of OTAC concentration on product morphology.

Figure S5. Histograms showing the lateral size distribution of crumpled Au nanosheets with different sizes.

Figure S6. Cross-viewed SEM images of crumpled Au nanosheets as displayed in Figure 4.

Figure S7. SEM images showing effect of alkyl chain length of capping agent on product morphology.

Figure S8. SEM images showing effect of reaction temperature on product morphology.

Figure S9. XRD pattern of flat Au nanosheets.

Figure S10. Histograms showing the lateral size distribution of flat Au nanosheets with different sizes.

Figure S11. Low-magnified SEM images of flat Au nanosheets with different sizes.

Figure S12. Cross-viewed SEM images of crumpled Au nanosheets as displayed in Figure 6.

Figure S13. Plots showing the relationship between lateral size and concentration of OTAC for the case of crumpled Au nanosheets and flat Au nanosheets.

Figure S14. SEM images showing effect of AA concentration on product morphology.

Figure S15. TEM images of CGNSs/C and FGNSs/C after CA measurements for 10000 seconds.

Table S1. Information of chemicals used in current work.

Table S2. Information of instruments used in current work.

Table S3. Comparison of the activities of typical Au electrocatalysts with various morphologies for EOR in alkaline media.

Details for electrochecmical messurements.

References 

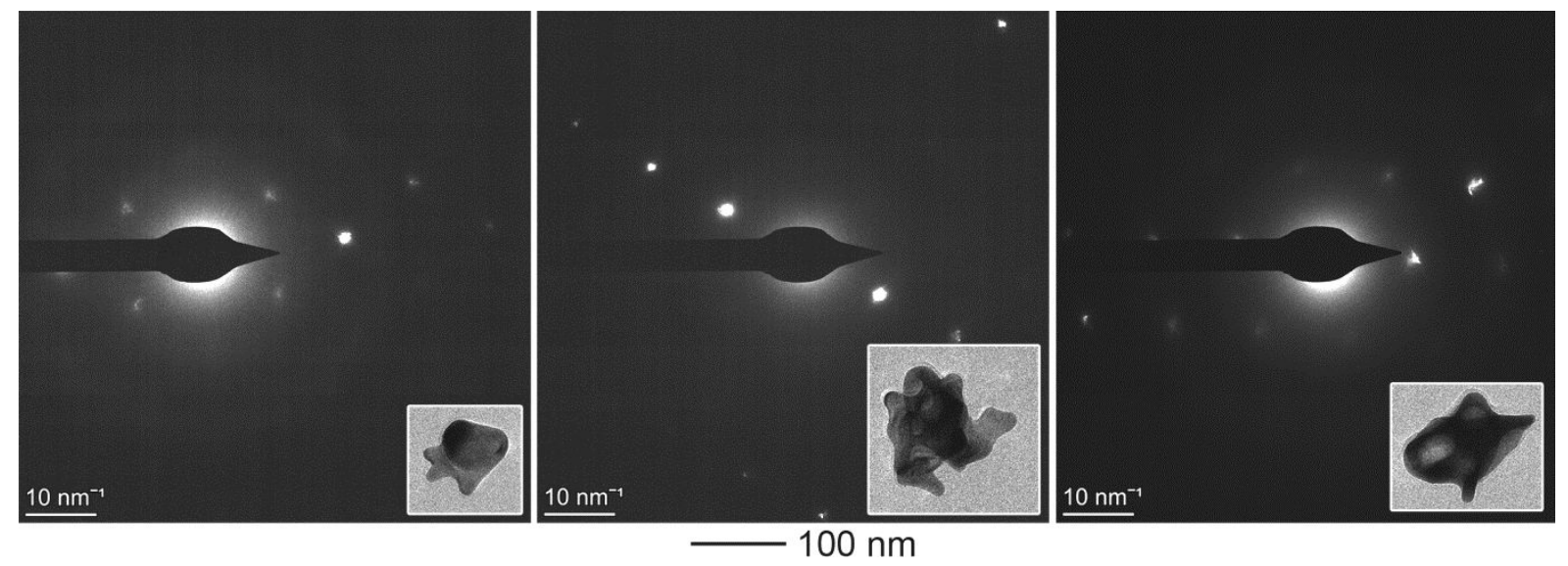

Figure S1. ED patterns of typical crumpled Au nanosheets. Corresponding particles are shown in the inset and the scale bar is shown at the bottom part. 

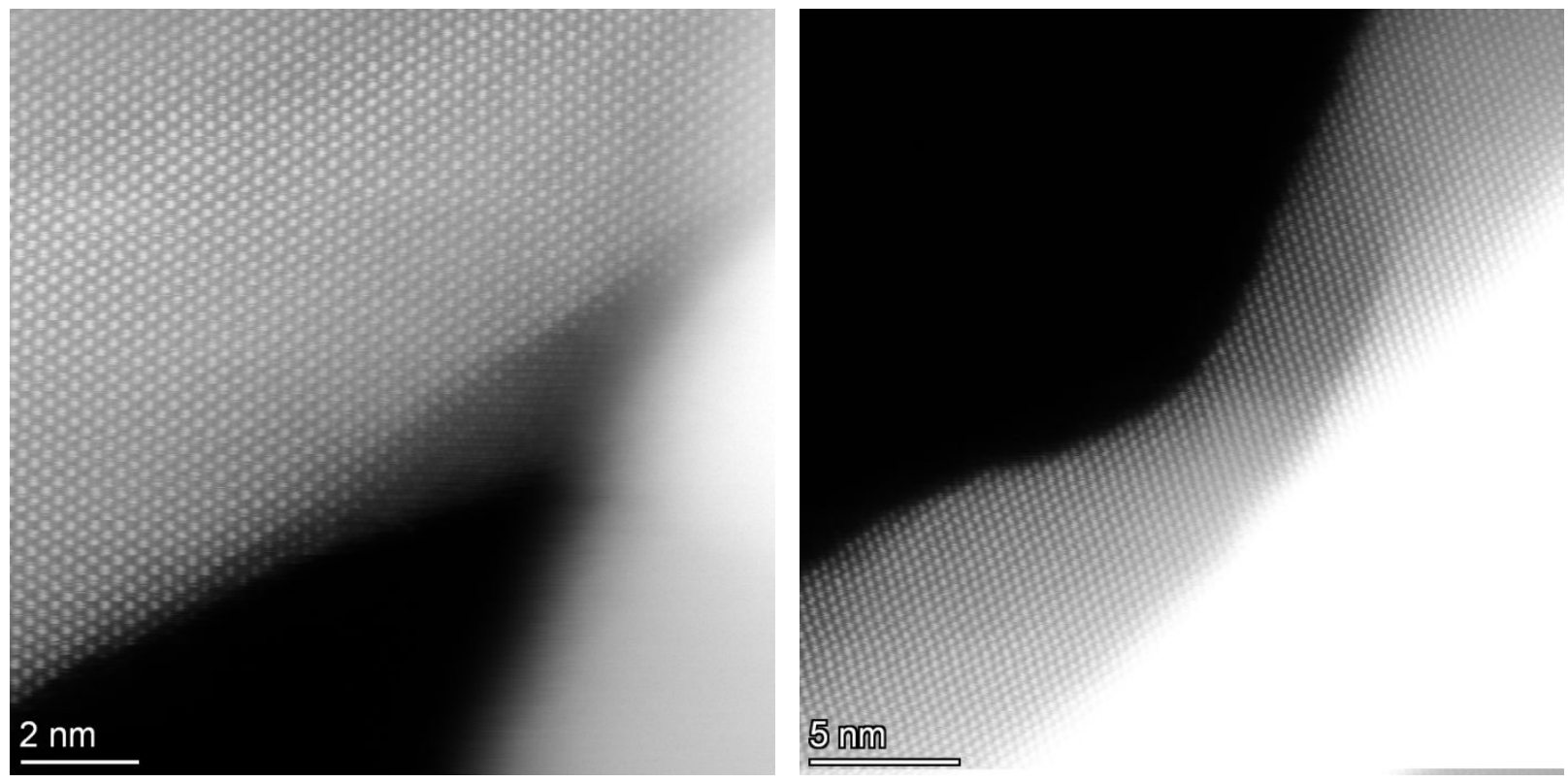

Figure S2. Atomic-resolution HAADF-STEM images of crumpled Au nanosheet showing the presence of stepped facets on edges. 

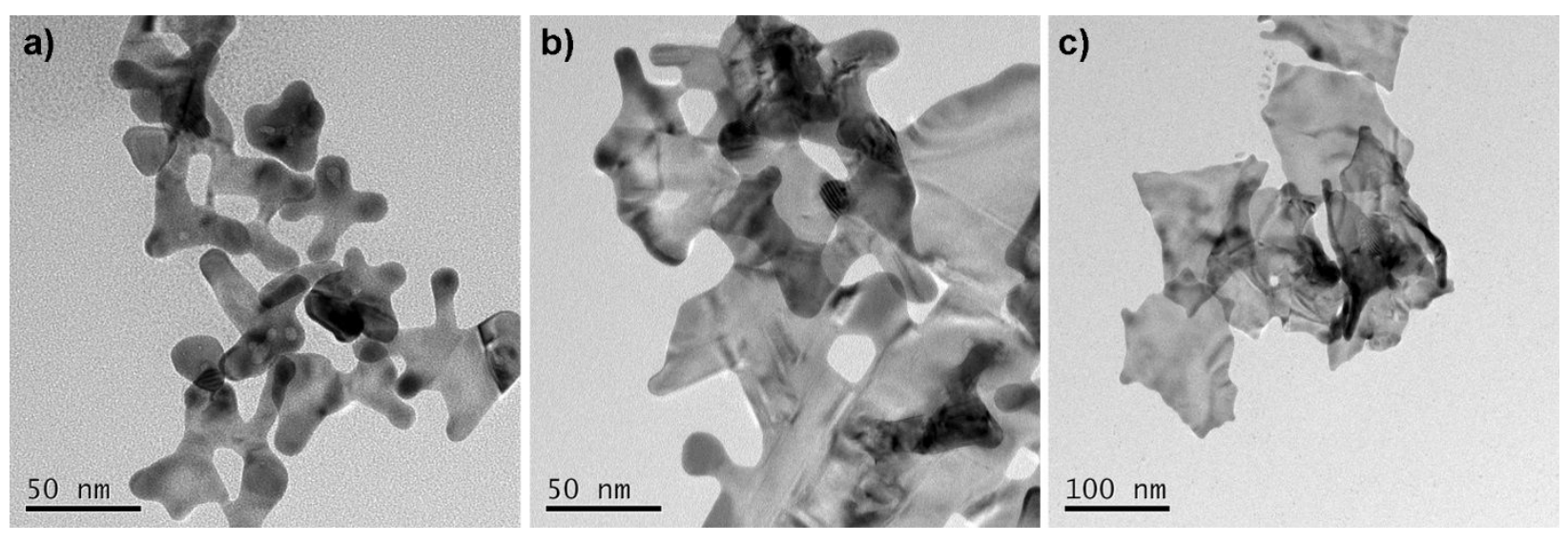

Figure S3. TEM images of Au products obtained via the standard procedure, except that the amount of Au precursor was reduced to a) $0.25 \mu \mathrm{mol}$, b) $0.50 \mu \mathrm{mol}$, and c) $1 \mu \mathrm{mol}$, respectively. 

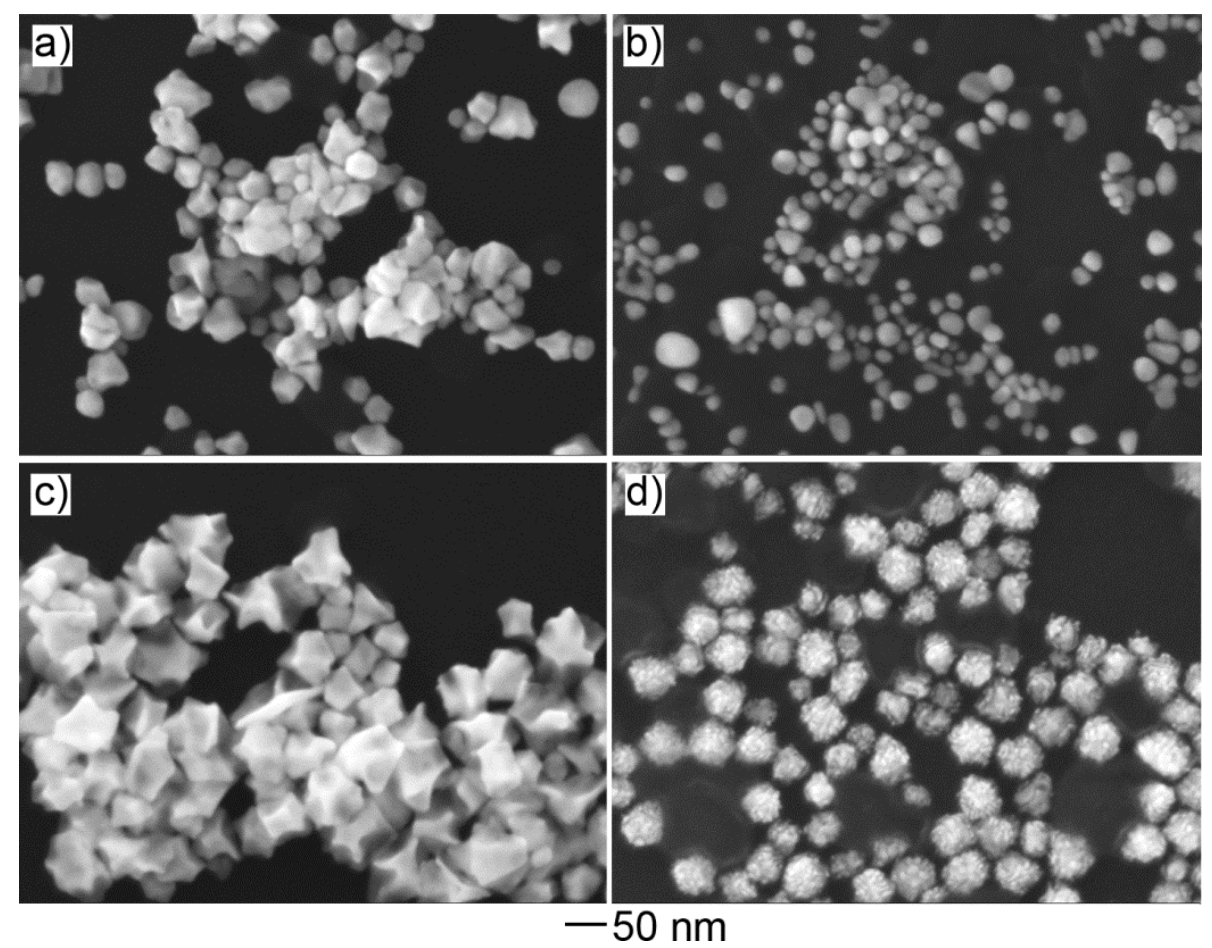

Figure S4. Effect of OTAC concentration on product morphology. SEM images of Au products obtained via the standard procedure, except that the concentration of OTAC was varied from 4.17 $\mathrm{mM}$ in standard procedure to: a) $0.42 \mathrm{mM}$; b) $2.1 \mathrm{mM}$; c) $16.7 \mathrm{mM}$; d) $41.7 \mathrm{mM}$, respectively. 

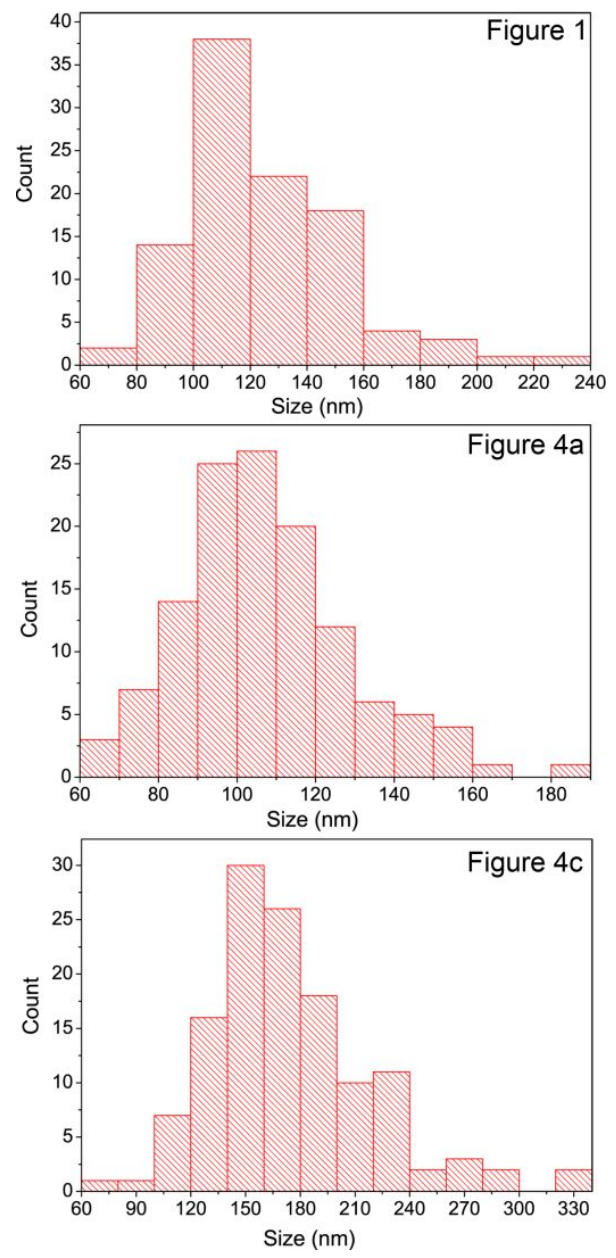

Figure S5. Histograms showing the lateral size distribution of crumpled $\mathrm{Au}$ nanosheets as displayed in current work. These data was obtained by statistically counting 100 typical nanosheets in corresponding low-magnified SEM images 

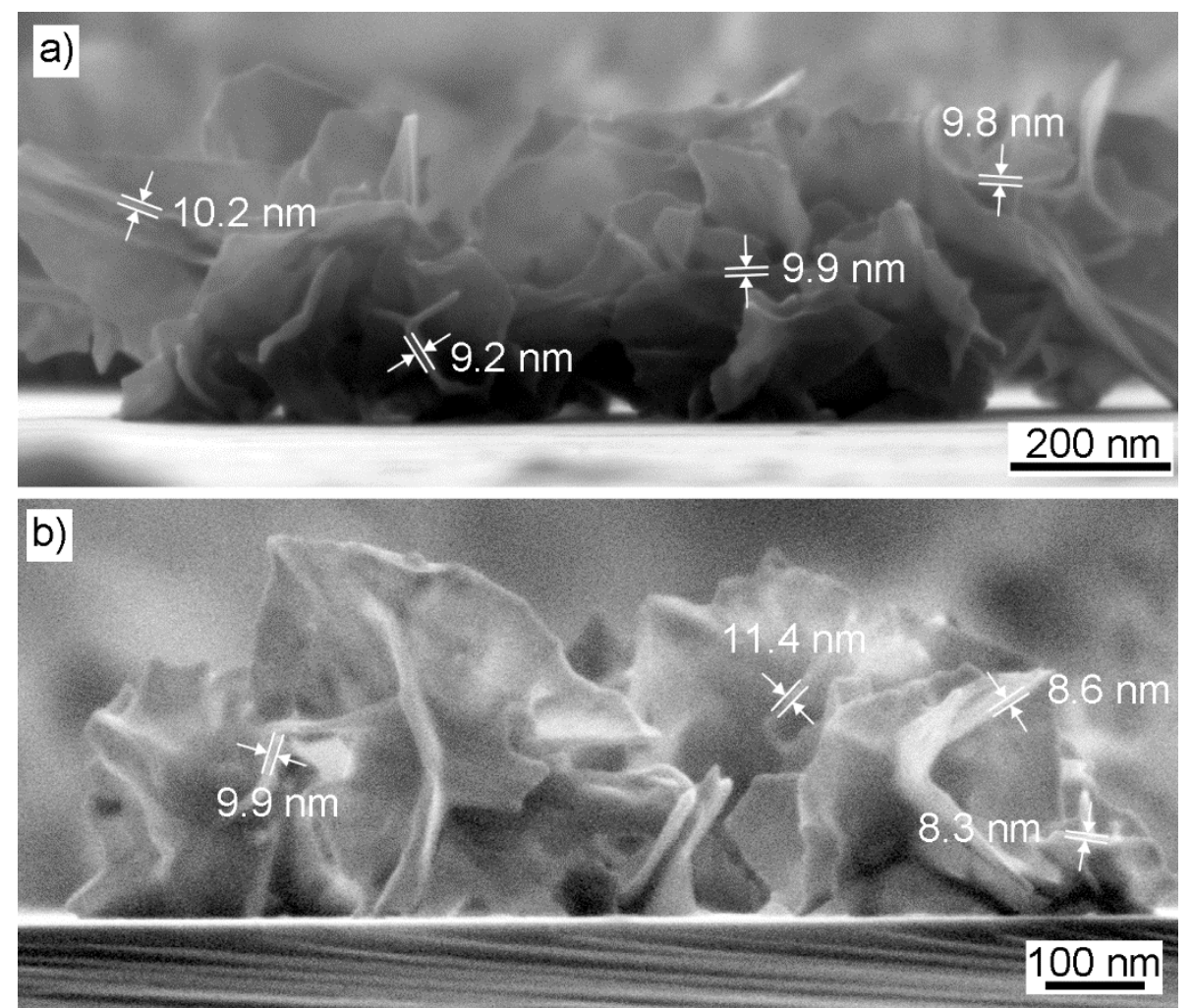

Figure S6. Cross-viewed SEM images of crumpled Au nanosheets as displayed in Figure 4: a)

Figure 4, a and b; b) Figure 4, $c$ and d. 

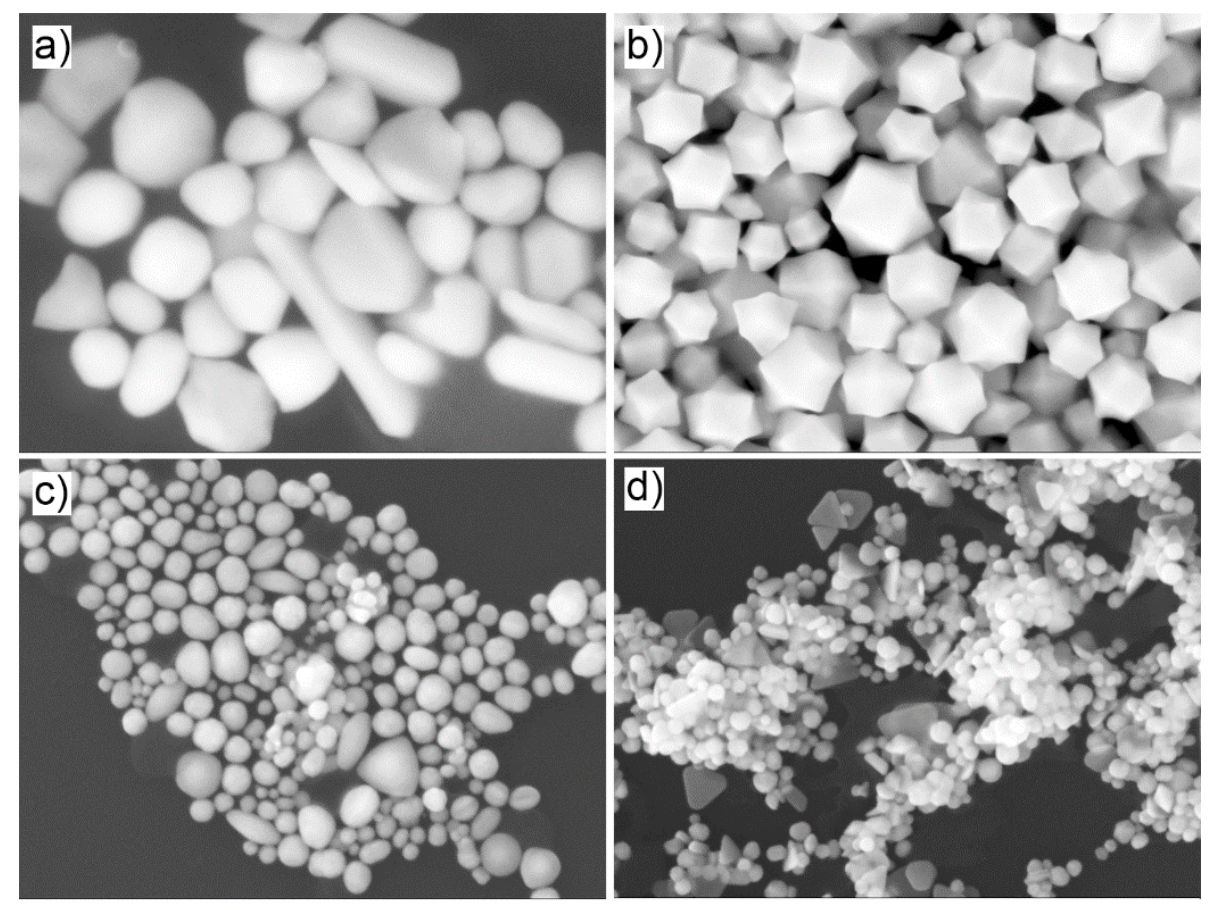

$100 \mathrm{~nm}$

Figure S7. Effect of alkyl chain length of capping agent on product morphology. SEM images of products obtained via the standard procedure, except that octadecyltrimethylammonium chloride (OTAC, $\mathrm{n}=18$ ) was replaced by a) docosyltrimethylammonium chloride (DCTAC, $\mathrm{n}=22$ ), b) cetyltrimethylammonium chloride $($ CTAC, $n=16), c)$ tetradecyltrimethylammonium chloride (TTAC, $\mathrm{n}=14$ ), d) dodecyltrimethylammonium chloride (DDTAC, $\mathrm{n}=12$ ), respectively, at the same concentration $(4.17 \mathrm{mM})$. 


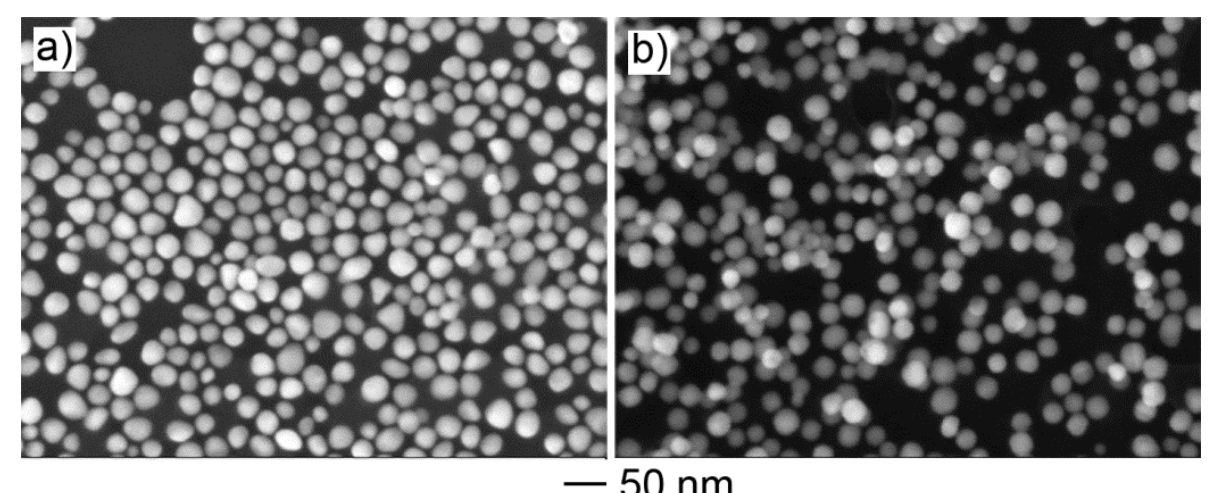

Figure S8. Effect of reaction temperature on product morphology. SEM images of Au products obtained via the standard procedure, except that the reaction temperature was set to a) 60 and b) $100{ }^{\circ} \mathrm{C}$, respectively. 


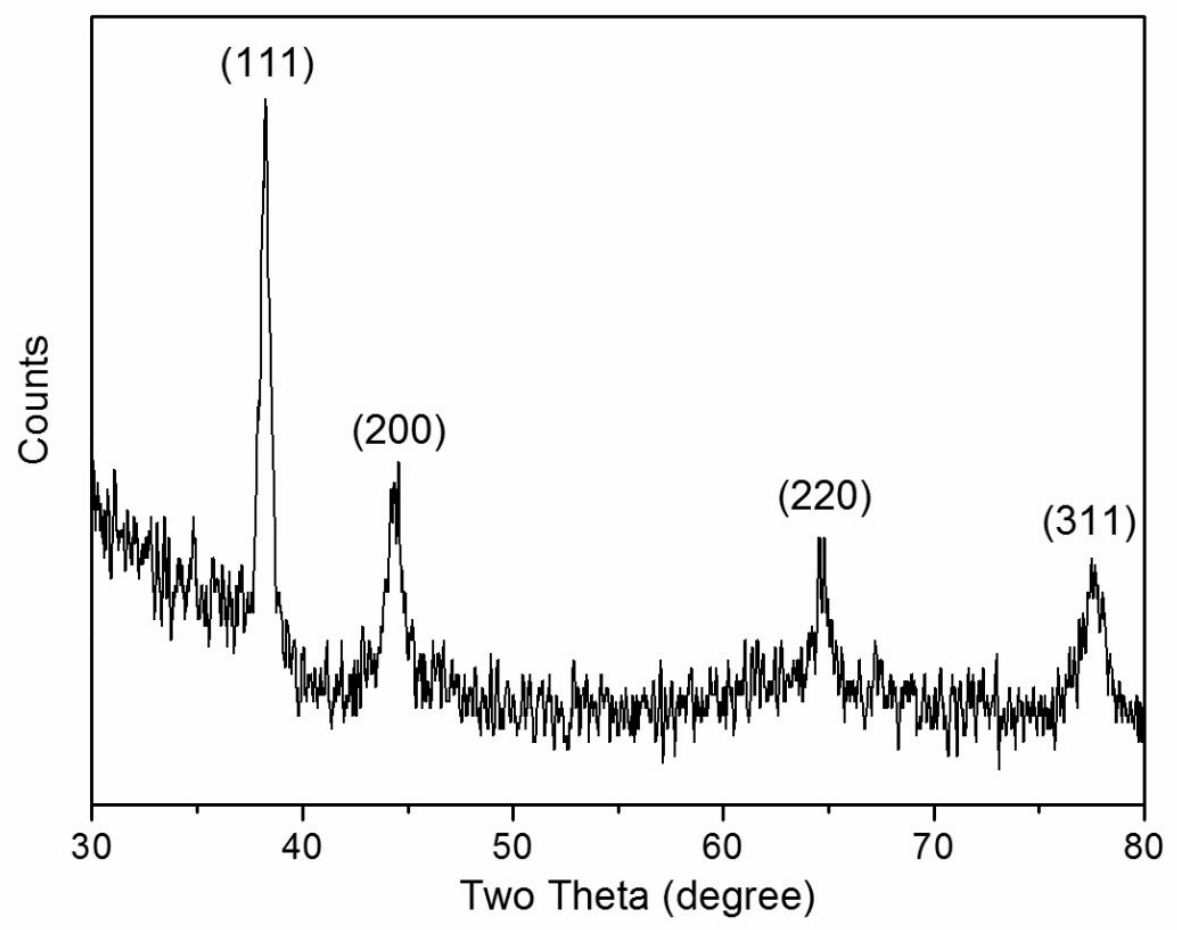

Figure S9. XRD pattern of flat Au nanosheets. 

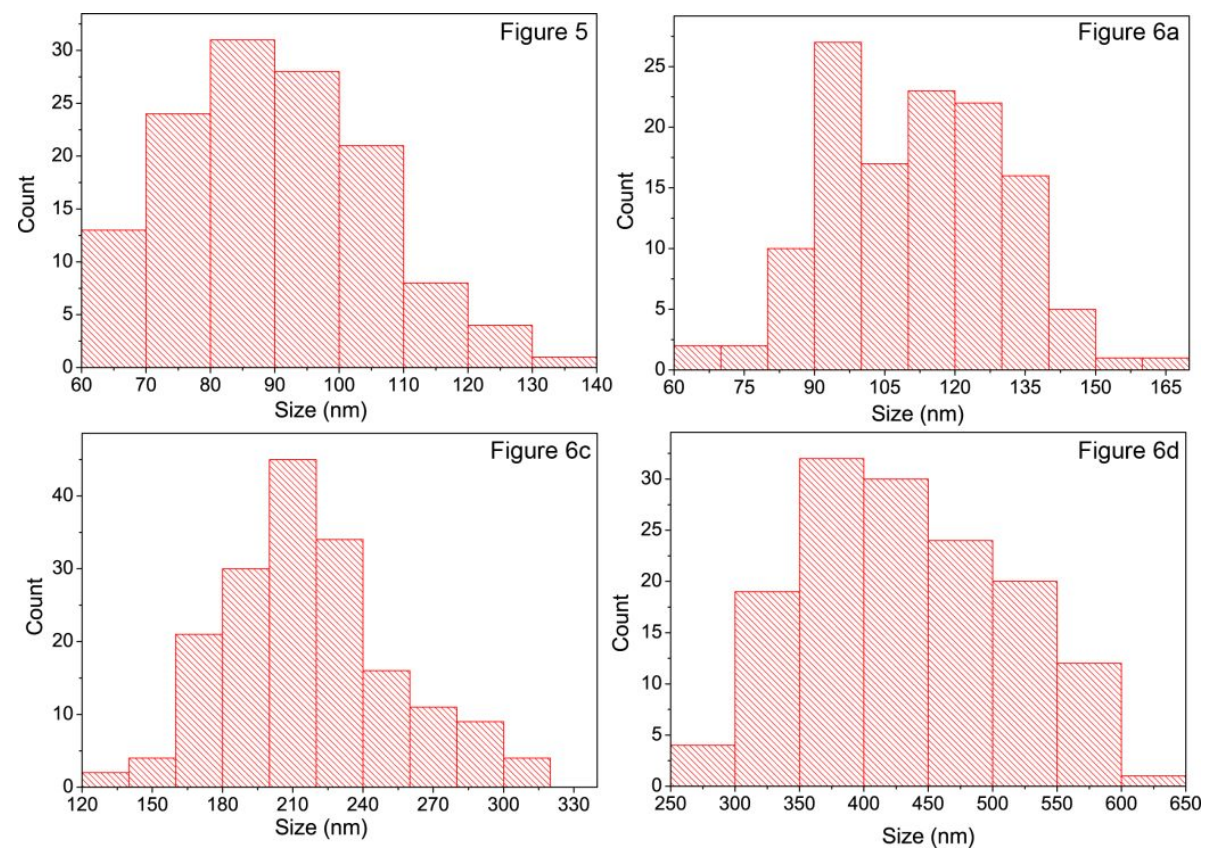

Figure S10. Histograms showing the lateral size distribution of flat Au nanosheets as displayed in current work. These data was obtained by statistically counting 100 typical nanosheets in corresponding low-magnified SEM images 


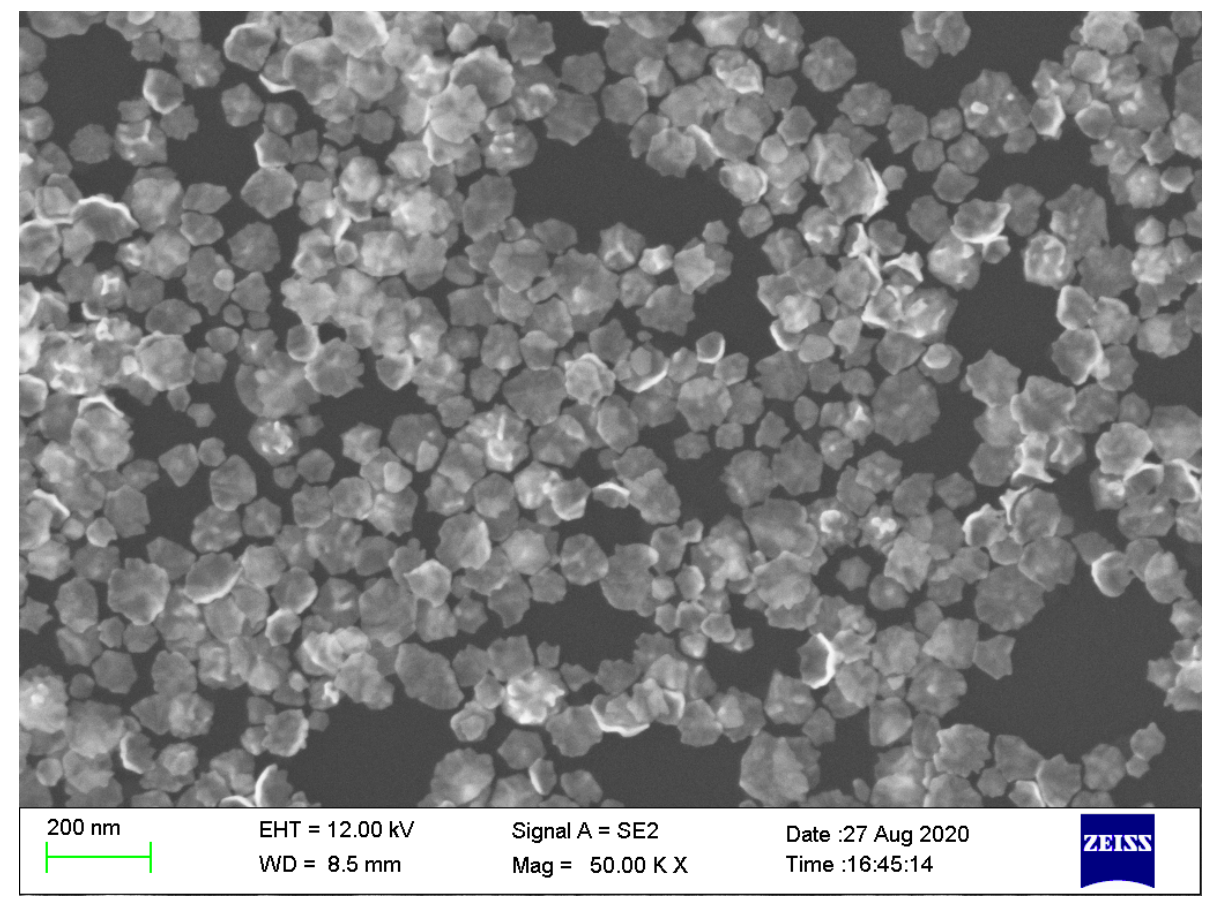

Figure S11a. Low-magnified SEM image of flat Au nanosheets as displayed in Figure 5.

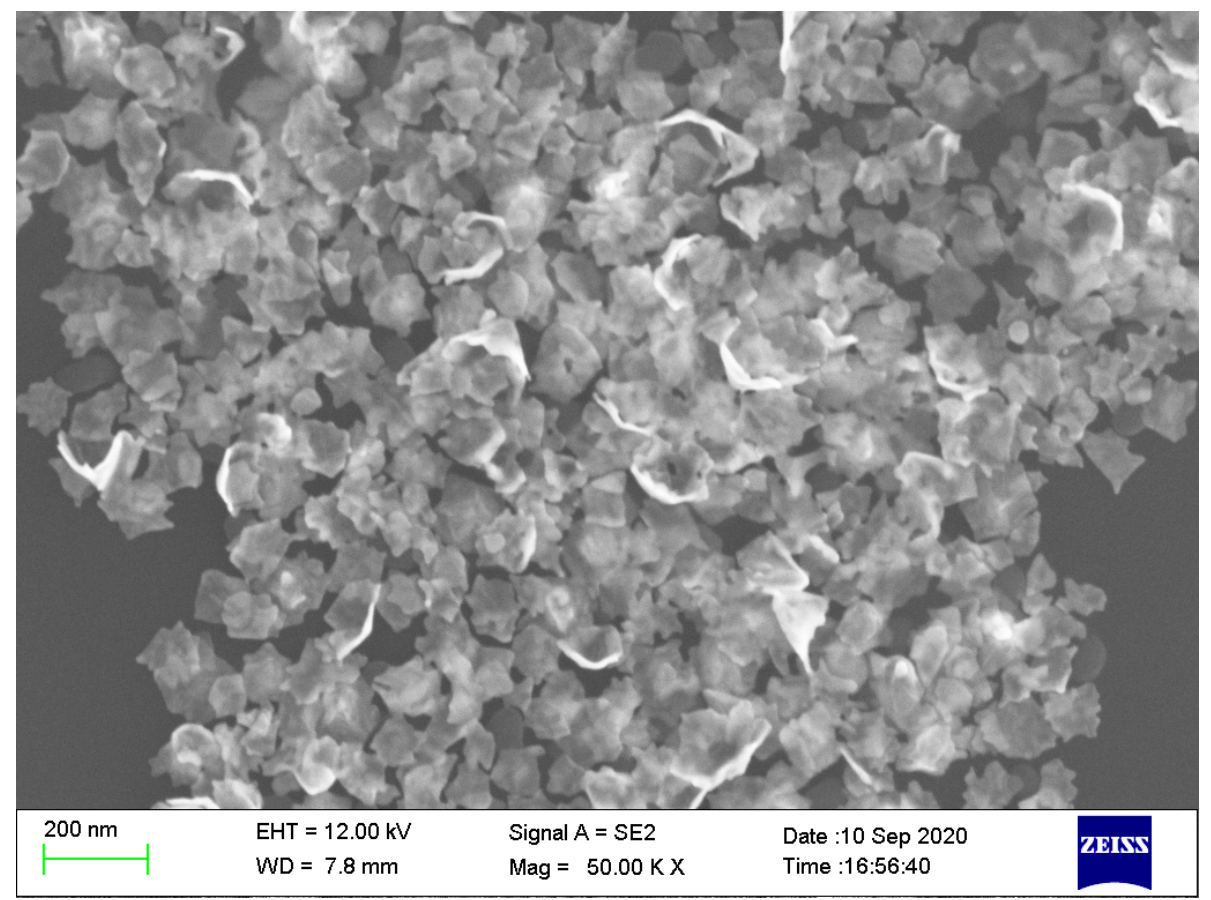

Figure S11b. Low-magnified SEM image of flat Au nanosheets as displayed in Figure 6a. 


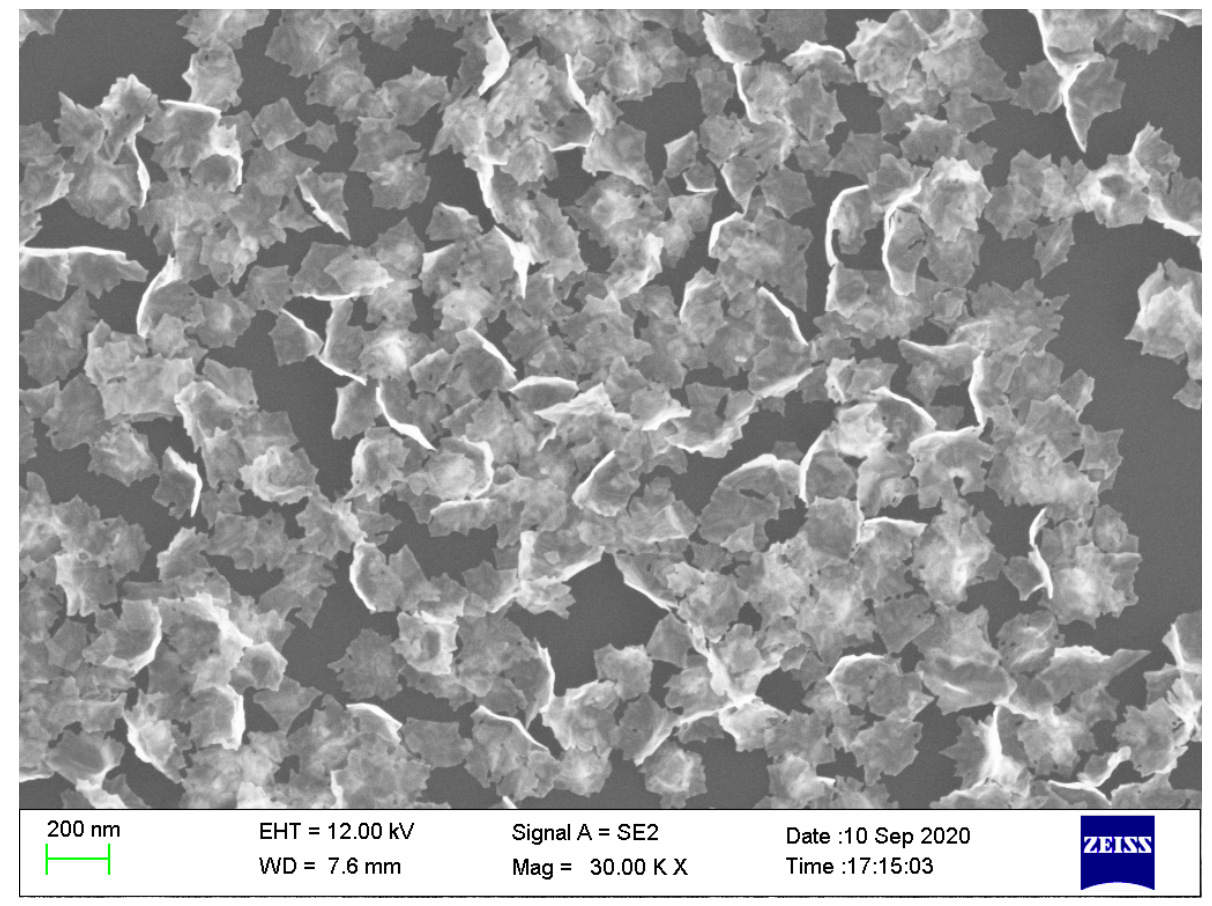

Figure S11c. Low-magnified SEM image of flat Au nanosheets as displayed in Figure 6c.

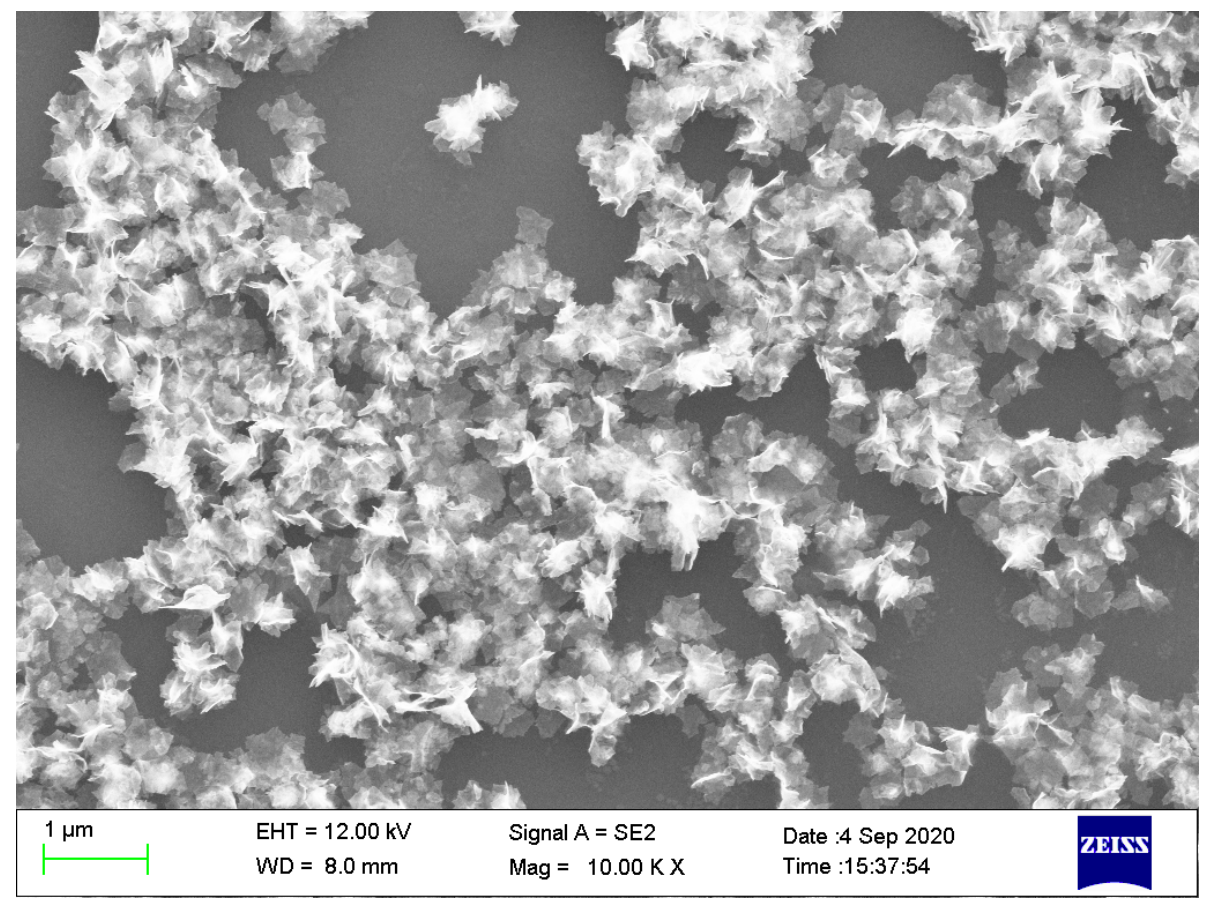

Figure S11d. Low-magnified SEM image of flat Au nanosheets as displayed in Figure 6d. 

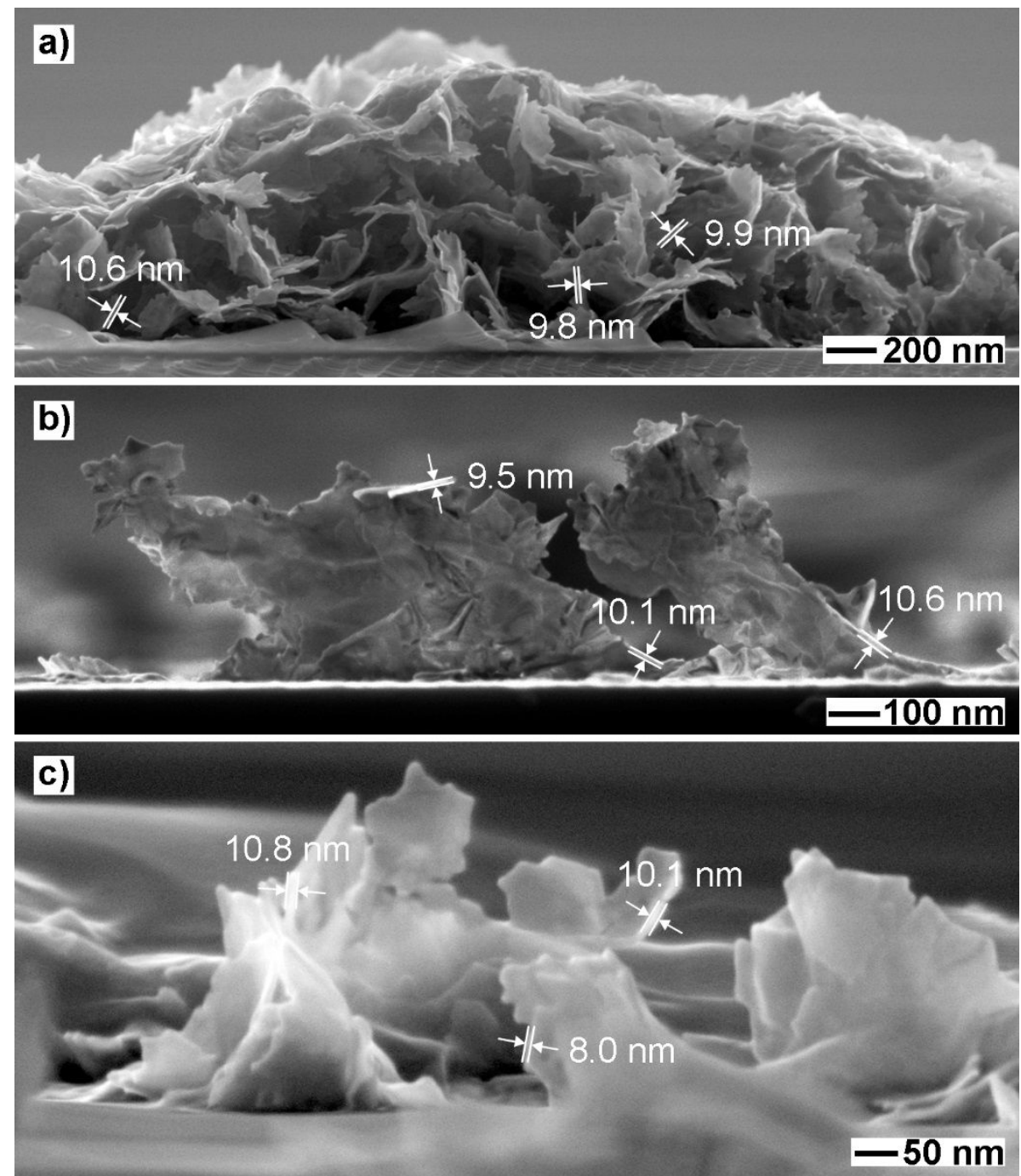

Figure S12. Cross-viewed SEM images of flat Au nanosheets as displayed in a) Figure 5, b) Figure 6c, and c) Figure 6d, respectively. 

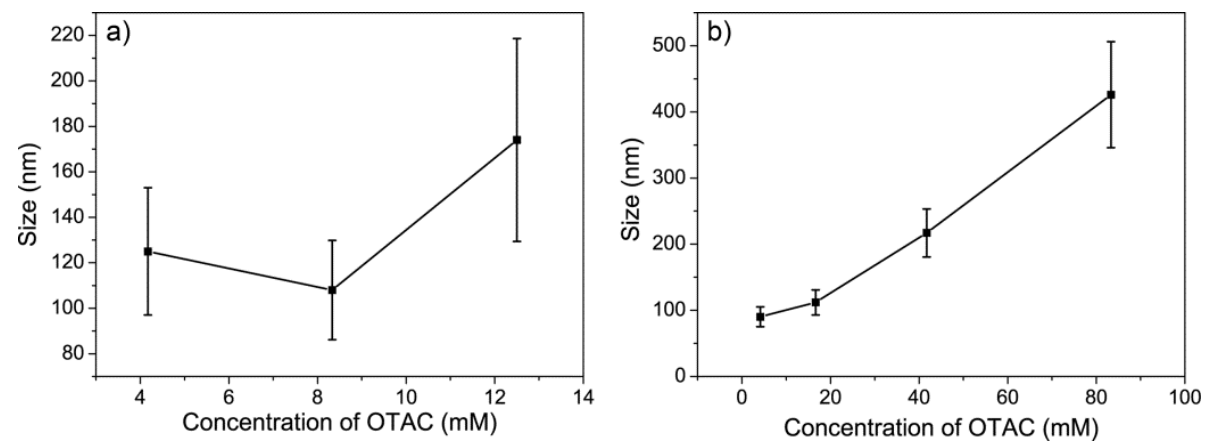

Figure S13. Plots showing the relationship between lateral size and concentration of OTAC for the case of a) crumpled Au nanosheets and b) flat Au nanosheets. 


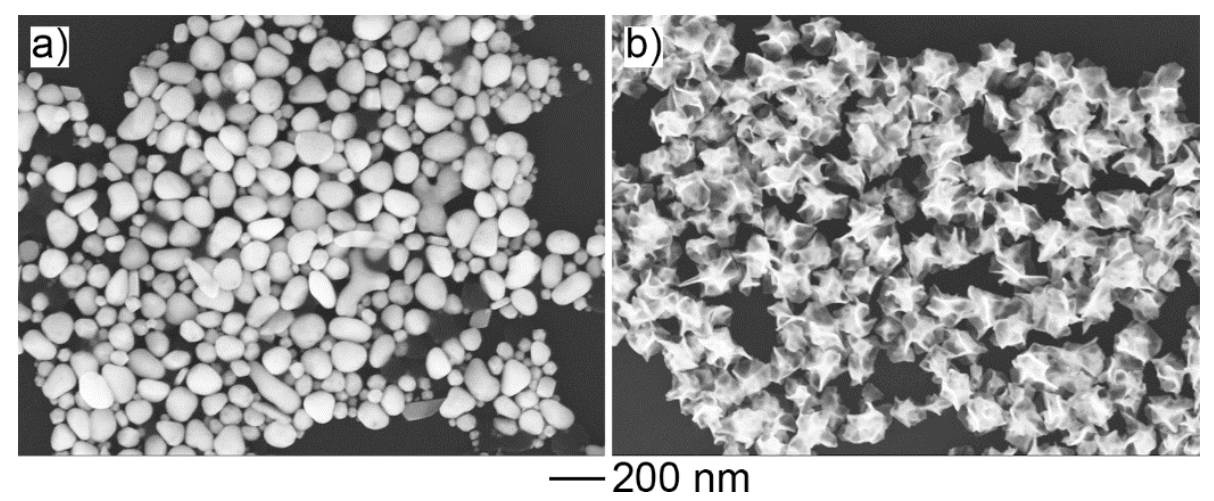

Figure S14. Effect of AA concentration on product morphology. SEM images of Au products obtained via the standard procedure, except that the AA concentration was varied from $0.83 \mathrm{mM}$ to a) $0.28 \mathrm{mM}$ and b) $8.33 \mathrm{mM}$, respectively. 

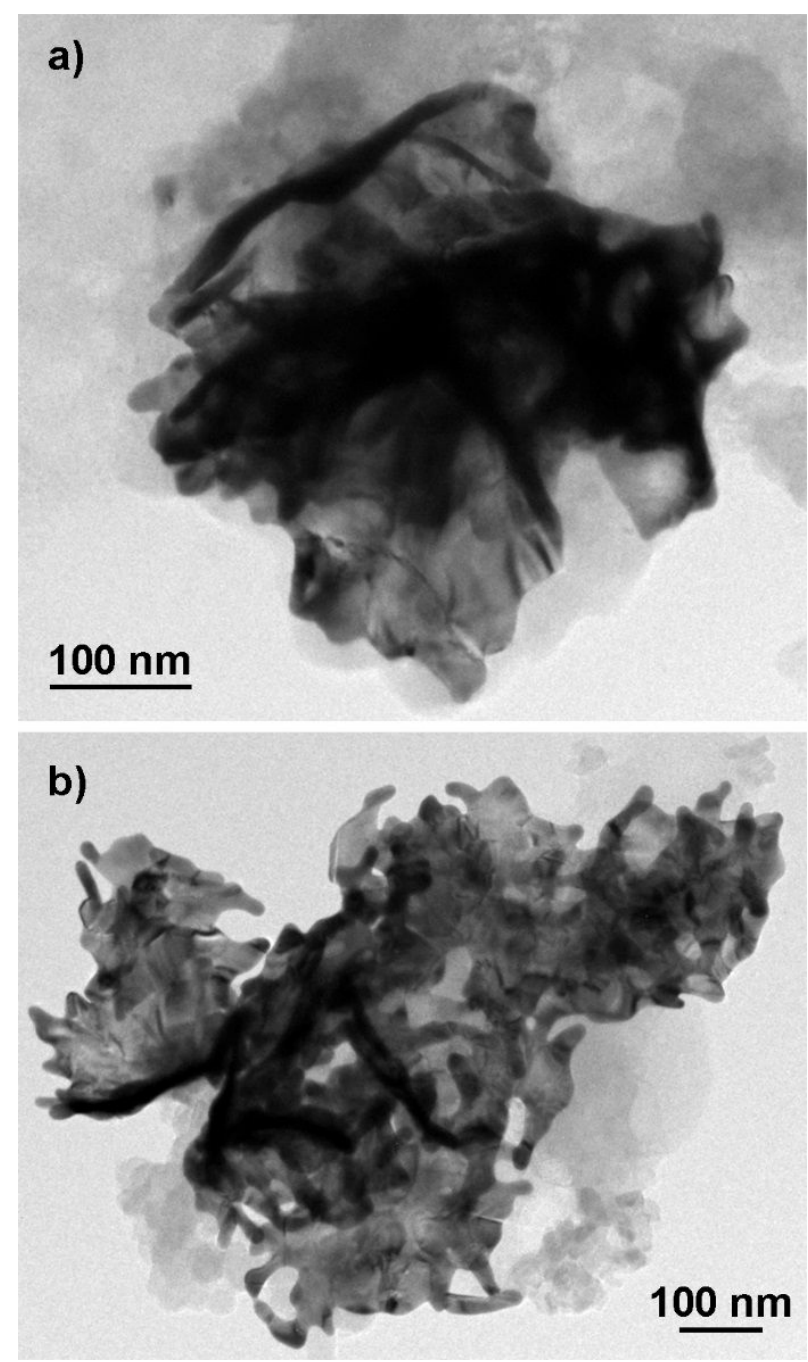

Figure S15. TEM images of a) CGNSs/C and b) FGNSs/C after CA measurements for 10000 seconds. 
Table S1. Information of chemicals used in current work.

\begin{tabular}{ccccc}
\hline Name & $\begin{array}{c}\text { Formula/Abbrev } \\
\text { iation/Model }\end{array}$ & Purity & Supplier & Location \\
\hline $\begin{array}{c}\text { gold(III) chloride } \\
\text { trihydrate } \\
\text { ascorbic acid } \\
\text { hexadecyltrimethyla } \\
\text { mmonium chloride }\end{array}$ & $\mathrm{HAuCl}_{4} \cdot 3 \mathrm{H}_{2} \mathrm{O}$ & $99.9 \%$ & & \\
$\begin{array}{c}\text { octadecyltrimethyla } \\
\text { mmonium chloride }\end{array}$ & $\mathrm{AA}$ & $99.0 \%$ & Aladdin & Shanghai, \\
docosyltrimethylam \\
$\begin{array}{c}\text { monium chloride } \\
\text { trimethyltetradecyla } \\
\text { mmonium chloride }\end{array}$
\end{tabular}

In all experiments, we used deionized water with a resistivity of $18.2 \mathrm{M} \Omega \cdot \mathrm{cm}$, which was prepared using an ultrapure water system (Ulupure, China). 
Table S2. Information of instruments used in current work.

\begin{tabular}{|c|c|c|c|c|c|}
\hline $\begin{array}{c}\text { Characterization } \\
\text { Technique }\end{array}$ & Abbreviation & $\begin{array}{l}\text { Instrument } \\
\text { Model No. }\end{array}$ & Condition & Brand & Location \\
\hline $\begin{array}{c}\text { Transmission } \\
\text { electron microscopy }\end{array}$ & TEM & & & & \\
\hline $\begin{array}{l}\text { high-resolution } \\
\text { TEM }\end{array}$ & HRTEM & Talos F200X & $200 \mathrm{kV}$ & FEI & USA \\
\hline Electron diffraction & ED & & & & \\
\hline $\begin{array}{l}\text { Scanning electron } \\
\text { microscopy }\end{array}$ & SEM & Ultra-60 & $12 \mathrm{kV}$ & Zeiss & Germany \\
\hline $\begin{array}{l}\text { High-angle annular } \\
\text { dark-field scanning } \\
\text { transmission } \\
\text { electron microscopy }\end{array}$ & $\begin{array}{l}\text { HAADF- } \\
\text { STEM }\end{array}$ & $\begin{array}{l}\text { spherical } \\
\text { aberration } \\
\text { (Cs)- } \\
\text { corrected } \\
\text { ARM-200F }\end{array}$ & $200 \mathrm{kV}$ & JEOL & Japan \\
\hline $\mathrm{X}$-ray diffraction & $\mathrm{XRD}$ & MiniFlex600 & l & Rigaku & Japan \\
\hline $\begin{array}{c}\text { UV-vis-NIR } \\
\text { extinction } \\
\text { spectroscopy }\end{array}$ & l & Lambda 750 & l & $\begin{array}{l}\text { Perkin } \\
\text { Elmer }\end{array}$ & USA \\
\hline $\begin{array}{l}\text { Inductively coupled } \\
\text { plasma optical } \\
\text { emission } \\
\text { spectroscopy }\end{array}$ & ICP-OES & ICAP-7200 & l & $\begin{array}{l}\text { Thermo } \\
\text { Fisher }\end{array}$ & USA \\
\hline
\end{tabular}


Table S3. Comparison of the activities of typical Au electrocatalysts with various morphologies for EOR in alkaline media.

\begin{tabular}{|c|c|c|c|c|c|c|c|}
\hline Electrocatalyst & Electrolyte & $\begin{array}{c}\text { Onset } \\
\text { Potential }\end{array}$ & $\begin{array}{l}\text { Oxidation } \\
\text { peak } \\
\text { potential }\end{array}$ & $\mathrm{j}_{\mathrm{r}} / \mathrm{j}_{\mathrm{f}}$ & ECSA & $\begin{array}{l}\text { Specific } \\
\text { Activity } \\
\left(\mathrm{mA} \mathrm{mg} \mathrm{Au}^{-}\right. \\
1)\end{array}$ & Ref. \\
\hline $\begin{array}{c}\text { “clean” Au } \\
\text { nanodendrites }\end{array}$ & $\begin{array}{l}0.5 \mathrm{M} \mathrm{NaOH} \\
+1 \mathrm{M} \text { ethanol }\end{array}$ & $\begin{array}{l}0.14 \mathrm{~V} \\
\text { vs. SCE }\end{array}$ & $\begin{array}{c}0.26 \mathrm{~V} \text { vs. } \\
\text { SCE }\end{array}$ & 0.12 & $\begin{array}{l}0.153 \mathrm{~cm}^{2} \text { (bare } \\
\left.\text { GCE: } 0.062 \mathrm{~cm}^{2}\right)\end{array}$ & / & 1 \\
\hline $\begin{array}{c}\text { dendritic Au } \\
\text { nanostructures with } \\
\text { hyperbranched } \\
\text { architectures }\end{array}$ & $\begin{array}{c}1 \mathrm{M} \mathrm{KOH} \\
+1 \mathrm{M} \text { ethanol }\end{array}$ & / & $\begin{array}{l}0.17 \mathrm{~V} \text { vs. } \\
\mathrm{Ag} / \mathrm{AgCl}\end{array}$ & / & $0.066 \mathrm{~cm}^{2}$ & 137.4 & 2 \\
\hline Au microdendrites & \multirow{2}{*}{$\begin{array}{c}1 \mathrm{M} \mathrm{NaOH} \\
+1 \mathrm{M} \text { ethanol }\end{array}$} & $\begin{array}{l}-0.04 \mathrm{~V} \\
\text { vs. SCE }\end{array}$ & $\begin{array}{c}0.16 \mathrm{~V} \text { vs. } \\
\text { SCE }\end{array}$ & 0.27 & / & / & \multirow{2}{*}{3} \\
\hline bulk Au & & $\begin{array}{l}-0.06 \mathrm{~V} \\
\text { vs. SCE }\end{array}$ & $\begin{array}{c}0.18 \text { V vs. } \\
\text { SCE }\end{array}$ & 0.64 & / & / & \\
\hline $\mathrm{Au} / \mathrm{C}$ & $\begin{array}{l}0.1 \mathrm{M} \mathrm{KOH} \\
+2 \mathrm{M} \text { ethanol }\end{array}$ & / & $\begin{array}{l}0.35 \mathrm{~V} \text { vs. } \\
\mathrm{Hg} \mid \mathrm{HgO}\end{array}$ & / & / & 442 & 4 \\
\hline $\begin{array}{l}\text { branched } \mathrm{Au} \\
\text { nanocorals }\end{array}$ & $\begin{array}{l}0.5 \mathrm{M} \mathrm{NaOH} \\
+1 \mathrm{M} \text { ethanol }\end{array}$ & $\begin{array}{c}\sim-0.15 \\
\text { V vs. } \\
\text { SCE }\end{array}$ & $\begin{array}{c}\sim 0.19 \mathrm{~V} \text { vs. } \\
\text { SCE }\end{array}$ & $\sim 0.43$ & $11.3 \mathrm{~m}^{2} \mathrm{~g}_{\mathrm{Au}}{ }^{-1}$ & $\sim 175$ & 5 \\
\hline nanoporous $\mathrm{Au}$ & \multirow{5}{*}{$\begin{array}{c}1 \mathrm{M} \mathrm{KOH} \\
+1 \mathrm{M} \text { ethanol }\end{array}$} & $\begin{array}{l}1.20 \mathrm{~V} \\
\text { vs. RHE }\end{array}$ & $\begin{array}{c}1.26 \mathrm{~V} \text { vs. } \\
\text { RHE }\end{array}$ & 0.24 & $13.0 \mathrm{~m}^{2} \mathrm{~g}_{\mathrm{Au}}{ }^{-1}$ & $308 \pm 3$ & \multirow{2}{*}{6} \\
\hline polycrystalline $\mathrm{Au}$ & & $\begin{array}{c}1.23 \mathrm{~V} \\
\text { vs. RHE }\end{array}$ & $\begin{array}{l}1.28 \mathrm{~V} \text { vs. } \\
\text { RHE }\end{array}$ & / & $10.0 \mathrm{~m}^{2} \mathrm{~g}_{\mathrm{Au}}{ }^{-1}$ & 22.1 & \\
\hline $\begin{array}{l}\text { Au nano-dendrites with } \\
\text { sub-10 nm arms }\end{array}$ & & $\begin{array}{c}-322 \\
\mathrm{mV} \text { vs. } \\
\text { SCE }\end{array}$ & $\begin{array}{c}270 \mathrm{mV} \text { vs. } \\
\text { SCE }\end{array}$ & 0.26 & $4.68 \mathrm{~m}^{2} \mathrm{~g}_{\mathrm{Au}}{ }^{-1}$ & 156.63 & 7 \\
\hline $\begin{array}{l}\text { crumpled } \mathrm{Au} \\
\text { nanosheets }\end{array}$ & & $\begin{array}{c}-361 \\
\mathrm{mV} \text { vs. } \\
\text { SCE }\end{array}$ & $\begin{array}{c}228 \mathrm{mV} \text { vs. } \\
\mathrm{SCE}\end{array}$ & 0.58 & $5.80 \mathrm{~m}^{2} \mathrm{~g}_{\mathrm{Au}}{ }^{-1}$ & 237.23 & current \\
\hline flat Au nanosheets & & $\begin{array}{l}-365 \\
\mathrm{mV} \text { vs. }\end{array}$ & $\begin{array}{c}265 \mathrm{mV} \text { vs. } \\
\text { SCE }\end{array}$ & 0.50 & $5.54 \mathrm{~m}^{2} \mathrm{~g}_{\mathrm{Au}}{ }^{-1}$ & 226.90 & \\
\hline
\end{tabular}




\begin{tabular}{|l|l|l|l|l|l|l|l|}
\hline & & SCE & & & & & \\
\hline
\end{tabular}




\section{Details for Electrochemical measurements}

Electrochemical experiments were carried out in a standard three-electrode system controlled by a $\mathrm{CHI}-760 \mathrm{E}$ potentiostat (CH Instruments, China). A glassy-carbon electrode (3 $\mathrm{mm}$ in diameter), a saturated calomel electrode (SCE), and a graphite rod were used as the working-, reference-, and counter-electrode, respectively. $1 \mathrm{mg}$ (in terms of Au mass) of metal catalyst and $2 \mathrm{mg}$ of carbon black were dispersed in $1 \mathrm{~mL}$ of water and vigorously sonicated for $30 \mathrm{~min}$ to form a uniform ink. Subsequently, $3 \mu \mathrm{L}$ of the catalyst ink was drop-cast onto the glassy carbon electrode and dried naturally, followed by dropping $3 \mu \mathrm{L}$ of Nafion solution and dried naturally. Before measurements, electro-catalysts were first activated in nitrogen-saturated aqueous $\mathrm{KOH}$ solution $(1 \mathrm{M})$ by CV cycling between -0.2 and $0.6 \mathrm{~V}$ versus SCE at a scan rate of $200 \mathrm{mV} \mathrm{s}^{-1}$ until the reproducible curves were obtained. $\mathrm{CV}$ curves in aqueous $\mathrm{KOH}$ solution (1 M) with ethanol $(1 \mathrm{M})$ were then collected between -0.4 and $0.6 \mathrm{~V}$ versus SCE at a scan rate $50 \mathrm{mV} \mathrm{s}^{-1}$. The chronoamperometric (CA) curves were recorded under a constant potential of $0.23 \mathrm{~V}$ versus SCE.

ECSA was estimated from the $\mathrm{CV}$ curve in aqueous $\mathrm{KOH}$ solution $(1 \mathrm{M})$ using the following equation:

$$
\mathrm{ECSA}=\frac{\mathrm{Q}_{\mathrm{AuO}}}{0.386 \mathrm{mC} \cdot \mathrm{cm}^{-2} \times \mathrm{m}_{\mathrm{Au}}}
$$

Where in Eq. 1, $\mathrm{Q}_{\mathrm{AuO}}$ was the charge by integrating the reduction peak area of $\mathrm{AuO}$ to $\mathrm{Au}$, $\mathrm{m}_{\mathrm{Au}}$ was the Au mass on the working electrode as determined by ICP-OES, and $0.386 \mathrm{mC} \mathrm{cm}^{-2}$ was the charge required for the reduction of AuO monolayer. ${ }^{6}$ 


\section{References}

1. Feng, J.-J.; Li, A.-Q.; Lei, Z.; Wang, A.-J., Low-Potential Synthesis of "Clean" Au Nanodendrites and Their High Performance toward Ethanol Oxidation. ACS Appl. Mater. Interfaces 2012, 4, (5), 2570-2576.

2. Huang, J.; Han, X.; Wang, D.; Liu, D.; You, T., Facile Synthesis of Dendritic Gold Nanostructures with Hyperbranched Architectures and Their Electrocatalytic Activity toward Ethanol Oxidation. ACS Appl. Mater. Interfaces 2013, 5, (18), 9148-9154.

3. Ye, W.; Yan, J.; Ye, Q.; Zhou, F., Template-Free and Direct Electrochemical Deposition of Hierarchical Dendritic Gold Microstructures: Growth and Their Multiple Applications. J. Phys. Chem. C 2010, 114, (37), 15617-15624.

4. Yan, S.; Gao, L.; Zhang, S.; Zhang, W.; Li, Y.; Gao, L., Synthesis of Au/C catalyst with high electrooxidation activity. Electrochim. Acta 2013, 94, 159-164.

5. Liu, Z.; Fu, G.; Tang, Y.; Sun, D.; Chen, Y.; Lu, T., A facile, one-pot synthesis of highly branched $\mathrm{Au}$ nanocorals and their enhanced electrocatalytic activity for ethanol oxidation. CrystEngComm 2014, 16, (36), 8576-8581.

6. Zhang, A.; Chen, Y.; Yang, Z.; Ma, S.; Huang, Y.; Richter, G.; Schützendübe, P.; Zhong, C.; Wang, Z., Enhanced Electrocatalytic Activities toward the Ethanol Oxidation of Nanoporous Gold Prepared via Solid-Phase Reaction. ACS Appl. Energy Mater. 2020, 3, (1), 336-343.

7. Zhang, G.; Liu, Z.; Fu, X.; Zhao, W.; Yin, X.; Niu, X.; Kong, R.; Qu, F.; Zheng, Y., Facile synthesis of branched $\mathrm{Au}$ nanocrystals with sub-10-nm arms and their applications for ethanol oxidation reaction. J. Nanopart. Res. 2021, 23, (1), 22. 